\title{
DER GROßE KURFÜRST FRIEDRICH WILHELM VON BRANDENBURG (1620-1688) ALS „MEHRFACHHERRSCHER“ DER FRÜHEN NEUZEIT
}

Michael Rohrschneider, Der Große Kurfürst Friedrich Wilhelm von Brandenburg (1620-1688). Studien zu einem frühneuzeitlichen Mehrfachherrscher, (Historische Forschungen 119, 2019).

2020 feiern wir den 400. Geburtstag des Großen Kurfürsten, einer Heldengestalt der borussischen Geschichtsschreibung. Die klassische Sicht auf den Monarchen ist in den letzten Jahrzehnten zunehmend revidiert worden. Vor allem der Bonner Frühneuzeithistoriker Ernst Opgenoorth zeigte wiederholt Grenzen und beschränkte Handlungsspielräume brandenburg-preuBischer Politik im 17. Jahrhundert auf. In dieser Tradition steht Michael Rohrschneider, derzeitiger Ordinarius für Neuere Geschichte in Bonn, der in mehreren Einzelstudien seit 1997 die Herrschaft des Großen Kurfürsten unter der leitenden Fragestellung frühneuzeitlicher Mehrfachherrschaft untersucht hat. Dafür eignet sich das Länderkonglomerat des Großen Kurfürsten ganz besonders, das sich von Kleve, Mark und Ravensberg, über Magdeburg, Halberstadt, die Mark Brandenburg und Hinterpommern bis nach Ost-Preußen erstreckte. Alle diese Herrschaften verfügten über ein eigenes Rechtssystem und eine eigene Verwaltung und waren nur durch die Person des Monarchen an die Dynastie der Hohenzollern gebunden.

Rohrschneider gruppiert seine Studien in vier Hauptkapitel. Nach einigen einleitenden Bemerkungen untersucht er 1. Strukturen und Probleme brandenburg-preußischer Mehrfachherrschaft, bevor er 2. leitende poli- 
tische Akteure dieser Mehrfachherrschaft beleuchtet. Ein 3. Hauptkapitel widmet sich der Außen- und Reichspolitik des Großen Kurfürsten, während er 4. die Ebene der historischen Rezeption der Herrschaft des Großen Kurfürsten betrachtet.

Das erste Hauptkapitel wiederum gliedert sich in zwei Teile, von denen der erste die eigene Wahrnehmung der Mehrfachherrschaft durch die Hohenzollern betrachtet. Zentrale Quellengrundlage hierfür sind die Testamente des Herrscherhauses (S. 36). In einem zweiten Teil vergleicht Rohrschneider die Kompositmonarchien Spanien und Brandenburg-Preußen, denen beispielweise der Aufbau eines stehenden Heeres zur Verteidigung ihres Länderkonglomerates gemeinsam war (S. 58).

Das zweite Hauptkapitel thematisiert Mehrfachloyalitäten und divergierende Interessen der führenden Berater des Großen Kurfürsten. Während Johann Georg von Anhalt-Dessau als Generalfeldmarschall und Statthalter der Mark Brandenburg dem Großen Kurfürsten verantwortlich war, habe er dennoch eigene Interessen als Landesfürst und Vorsteher des Hauses Anhalt vertreten (S. 65). Reichspatriotisch, kaisertreu und frankreichfeindlich habe er zudem eine Politik verfolgt, die nicht immer im Einklang mit der Bündnispolitik seines Dienstherrn stand (S. 76). Mehrfachloyalitäten seien ebenso für Johann-Moritz von Nassau-Siegen in seiner Doppelrolle als niederländischer Feldmarschall und brandenburgischer Statthalter von Kleve, Mark und Ravensberg charakteristisch gewesen (S. 85f.). Durch verwandtschaftliche Beziehungen und ihre gemeinsame reformierte Konfession seien die beiden Statthalter mit dem Kurfürsten verbunden gewesen (S. 89f.). Daneben würden diese Fälle aber aufzeigen, dass ihre Würde als Reichsfürsten und ihre ausländischen Beziehungen auch ein politisches Kapital im Dienste Brandenburgs darstellten (bspw. S. 90, 98, 102). Über ein solches Potential verfügten landsässige Minister wie Otto von Schwerin nicht. Sein Verhältnis zum Großen Kurfürsten habe vor allem auf gleichem Bekenntnis (S. 106), Intelligenz und persönlicher Treue beruht (S. 117f.).

Alle drei behandelten Akteure waren auch auf dem Feld der Reichs- und Außenpolitik aktiv, der das dritte Hauptkapitel Rohrschneiders Darstellung gewidmet ist. Er behandelt am Beispiel Johann Georgs II. von Anhalt-Dessau die Praxis der Erstellung politischer Gutachten, welche die Grundlage der außenpolitischen Entscheidungsfindung am brandenburg-preußischen Hof bildeten. Die beiden von Rohrschneider behandelten Gutachten finden 
sich sauber ediert im Band (S. 130-132, 135-138). Eine zentrale Frage, die bereits die borussische Geschichtsschreibung aufgeworfen hat, ist außerdem diejenige nach dem Verhältnis des Großen Kurfürsten zum Haus Habsburg. Zusammen mit dem österreichischen Historiker Stefan Sienell kontrastiert Rohrschneider das Verhältnis beider Mächte im Alten Reich. Sie kommen zu dem Ergebnis, dass der Große Kurfürst einen Beitrag zur Stabilisierung der "traditionellen hierarchischen Ordnung“ im Alten Reich geleistet habe. Aufgrund geringer eigener Ressourcen sei er auf die Kooperation mit Kaiserhof angewiesen gewesen (S. 151). Ein dritter Aufsatz über die brandenburg-preußischen Statthalter als außenpolitische Akteure schließt das Kapitel ab. Ihre diplomatische Verwendung zeige, dass die Außenpolitik in der Hohenzollernschen Kompositmonarchie transterritorial ausgerichtet gewesen sei (S. 179).

Das vierte und letzte Hauptkapitel schließlich widmet sich anhand zweier Fallbeispiele der historiographischen Rezeption der Mehrfachherrschaft des Großen Kurfürsten. Ein erster Beitrag stellt die Kölner Antrittsvorlesung des Autors dar, in der er sich mit dem Herrscherbild Friedrich Wilhelms und seines Vaters Georg Wilhelms in den Mémoires pour servir à l'histoire de la maison de Brandenbourg Friedrichs des Großen auseinandersetzt. Hierbei betont er den legitimatorischen Charakter der Geschichtsschreibung Friedrichs II., der in anachronistischer Weise Parallelen zwischen seiner eigenen und der Politik seines Urgroßvaters gezogen habe (S. 205). Der zweite Teil seiner historiographiegeschichtlichen Ausführungen widmet sich der Rolle der Pommernpolitik des Großen Kurfürsten in der Geschichtsschreibung. Als abschließendes Urteil stellt der Autor Mehrfachherrschaft als historisches Beispiel für die Spannung zwischen „Einheit und regionaler Vielfalt“ in Europa dar (S. 223).

Jeder Aufsatz für sich ist klar strukturiert, fußt zu großen Teilen auf unveröffentlichten Archivalien und ist mit der für Michael Rohrschneider charakteristischen Präzision und Sauberkeit ausgearbeitet. Der Titel Studien zu einem frühneuzeitlichen Mehrfachherrscher und der unveränderte Abdruck der einzelnen Beiträge, den der Autor in der Einleitung selbst einräumt (S. 17), führt allerdings dazu, dass der Band nicht immer stringent dem Thema der Mehrfachherrschaft Friedrich Wilhelms folgt. Das ist bspw. bei den durchaus lesenswerten Ausführungen zur Innenpolitik Johann Georgs II. von Anhalt-Dessau (S. 66-69) oder der Behandlung Georg Wilhelms in der Geschichtsschreibung Friedrichs des Großen (S. 184) der Fall. 
Demgegenüber stehen vergleichende Studien zum Fürstentum Halberstadt und den Herzogtümern Magdeburg und Preußen für eine systematische Untersuchung brandenburg-preußischer Mehrfachherrschaft immer noch aus. Dies war freilich nicht Anspruch des zu besprechenden Bandes. Er stellt hierfür aber inhaltliche Grundlagenarbeit und ein methodisches Gerüst zur Verfügung, das auch nach dem Jubiläumsjahr den Charakter eines Grundlagenwerkes zur Herrschaft des Großen Kurfürsten und frühneuzeitlicher Mehrfachherrschaft allgemein beanspruchen kann. 